ANNALES

POLONICI MATHEMATICI

LXXIX.1 (2002)

\title{
Asymptotics for quasilinear elliptic non-positone problems
}

\author{
by ZuODONG YANG and QIShaO Lu (Beijing)
}

\begin{abstract}
In the recent years, many results have been established on positive solutions for boundary value problems of the form

$$
\begin{gathered}
-\operatorname{div}\left(|\nabla u(x)|^{p-2} \nabla u(x)\right)=\lambda f(u(x)) \quad \text { in } \Omega, \\
u(x)=0 \quad \text { on } \partial \Omega,
\end{gathered}
$$

where $\lambda>0, \Omega$ is a bounded smooth domain and $f(s) \geq 0$ for $s \geq 0$. In this paper, a priori estimates of positive radial solutions are presented when $N>p>1, \Omega$ is an $N$-ball or an annulus and $f \in C^{1}(0, \infty) \cup C^{0}([0, \infty))$ with $f(0)<0$ (non-positone).
\end{abstract}

1. Introduction. In this paper, we consider the set of positive radial solutions to the following boundary value problem for a quasilinear elliptic P.D.E.:

$$
\begin{gathered}
\operatorname{div}\left(|\nabla u|^{p-2} \nabla u\right)+\lambda f(u)=0 \quad \text { in } \Omega, \\
u=0 \quad \text { on } \partial \Omega,
\end{gathered}
$$

where $\Omega$ denotes an annulus or a ball in $\mathbb{R}^{N}(N>p>1)$, and $\lambda>0$.

The problem (1.1)-(1.2) arises in the theory of quasiregular and quasiconformal mappings or in the study of non-Newtonian fluids. In the latter case, the quantity $p$ is a characteristic of the medium. Media with $p>2$ are called dilatant fluids and those with $p<2$ are called pseudoplastics (see $[1-2])$. When $p \neq 2$, the problem becomes more complicated since certain nice properties inherent to the case $p=2$ seem to be invalid or at least difficult to verify. The main differences between $p=2$ and $p \neq 2$ are discussed in $[6,8]$. The existence and uniqueness of positive solutions of (1.1)-(1.2) have been studied by many authors, for example, $[4-10,13-21]$ and the references therein.

2000 Mathematics Subject Classification: Primary 35J25, 35J65.

Key words and phrases: elliptic, quasilinear, estimates, asymptotics.

Supported by National Science Foundation of China (No. 10172011) and Doctoral Foundation of National Educational Committee. 
By a positive solution $u$ of (1.1)-(1.2), we mean a function $u \in C_{0}^{1}(\Omega)$ with $u>0$ in $\Omega$ which satisfies

$$
\int_{\Omega}|\nabla u|^{p-2} \nabla u \nabla v=\lambda \int_{\Omega} f(u) v
$$

for any $v \in C_{0}^{\infty}(\Omega)$. Thus, these solutions are considered in a weak sense. By a small solution $u_{\lambda}$ of (1.1)-(1.2), we mean that $\lim _{\lambda \rightarrow 0^{+}}\left\|u_{\lambda}\right\|_{\infty}=0$ (or $\left.\lim _{\lambda \rightarrow \infty}\left\|u_{\lambda}\right\|_{\infty}=0\right)$. By a large positive solution $u_{\lambda}$ of (1.1)-(1.2), we mean that $\lim _{\lambda \rightarrow 0^{+}}\left\|u_{\lambda}\right\|_{\infty}=\infty$ (or $\left.\lim _{\lambda \rightarrow \infty}\left\|u_{\lambda}\right\|_{\infty}=\infty\right)$.

When $f$ is strictly increasing on $\mathbb{R}^{+}, f(0)=0, \lim _{s \rightarrow 0^{+}} f(s) / s^{p-1}=0$ and $f(s) \leq \alpha_{1}+\alpha_{2} s^{\mu}$, where $0<\mu<p-1$ and $\alpha_{1}, \alpha_{2}>0$, it has been shown in [6] that there exist at least two positive solutions for (1.1)-(1.2) when $\lambda$ is sufficiently large. If $\liminf _{s \rightarrow 0^{+}} f(s) / s^{p-1}>0, f(0)=0$ and the monotonicity hypothesis $\left(f(s) / s^{p-1}\right)^{\prime}<0$ holds for all $s>0$, it has been proved in [8] that the problem (1.1)-(1.2) has a unique positive solution when $\lambda$ is sufficiently large. If $f(s)>0$ for $s \geq 0$ and $\lim \sup _{s \rightarrow 0^{+}}\left(f(s) / s^{p-2}\right)^{\prime}<0$, it has been proved in [9] that the problem (1.1)-(1.2) has a unique small solution when $\lambda$ is sufficiently small. It also has been proved that there exists at least one large positive radial solution of (1.1)-(1.2) for $\Omega$ being an $N$-ball or an annulus when $\lambda$ is sufficiently small. If $f(0)<0$, related results have been obtained in $[7,20]$.

A natural question is to determine how $\lambda$ and $d=\max _{\Omega} u(\cdot, \lambda)=$ $\|u(\cdot, \lambda)\|_{\infty}$ are related. When $p=2, f(0)<0$ or $f(0)=0$ and $\Omega$ is a unit ball in $\mathbb{R}^{N}$, the related results have been obtained by $[11,12]$. In [21], the author studied this problem for the case where $\Omega$ is a unit ball in $\mathbb{R}^{N}$ and $f(0)<0, p>1$. In this paper, we further study this problem for $\Omega$ being an $N$-ball $(N>p>1)$ or an annulus and $f(0)<0$ (non-positone). This extends and complements previous results in the literature [11, 12, 21].

Consider a positive radial solution $u$ of (1.1)-(1.2); thus $u=u(r, \lambda)$ satisfies

$$
\left(r^{N-1}\left|u^{\prime}\right|^{p-2} u^{\prime}\right)^{\prime}+\lambda r^{N-1} f(u)=0 .
$$

If $\Omega$ is an annulus $0<r_{1} \leq r \leq r_{2}$, we introduce the transformation of variables

$$
s=r^{(p-N) /(p-1)}, \quad u(r)=v(s) .
$$

Thus (1.3) becomes

$$
\left(\left|v^{\prime}(s)\right|^{p-2} v^{\prime}(s)\right)^{\prime}+\lambda((p-1) /(N-p))^{p} s^{-p(N-1) /(N-p)} f(v(s))=0
$$

and the boundary conditions become

$$
v\left(s_{1}\right)=0, \quad v\left(s_{2}\right)=0 .
$$


If $\Omega=B_{1}(0)$, the boundary condition (1.2) becomes

$$
u^{\prime}(0)=0, \quad u(1)=0 .
$$

2. A priori estimates for $\Omega$ being an annulus. In this section, we consider the set of radially symmetric positive solutions to the equation

$$
\begin{cases}-\operatorname{div}\left(|\nabla u|^{p-2} \nabla u\right)=\lambda f(u) & \text { in } \Omega, \\ u=0 & \text { on } \partial \Omega,\end{cases}
$$

where $\Omega$ denotes an annulus in $\mathbb{R}^{N}(N>p>1)$ and $\lambda>0$. Here $f$ : $[0, \infty) \rightarrow \mathbb{R}$ satisfies the following assumptions:

(A) $f \in C^{1}(0, \infty) \cap C([0, \infty)), f(0)<0$, and there exists $\alpha>0$ such that $f(s)<0$ for $0<s<\alpha, f(\alpha)=0, f$ is increasing for $s>\alpha$ and $\lim _{s \rightarrow \infty} f(s)=\infty$.

(B) There are constants $L_{0}>0$ and $p-1<q<((p-1) N+p) /(N-p)$ such that $\lim _{u \rightarrow \infty} f(u) / u^{q}=L_{0}$.

Theorem 2.1. Suppose that conditions (A) and (B) hold. Then there exist positive constants $K_{1}$ and $K_{2}$ such that for small $\lambda$,

$$
K_{1}<\lambda\|u(\cdot, \lambda)\|_{\infty}^{q-p+1}<K_{2},
$$

where $\left\{u(\cdot, \lambda) \mid \lambda \in\left(0, \lambda_{0}\right)\right\}$ is an arbitrary positive radially symmetric solution of (1.1)-(1.2). Furthermore, for any sequence $\left\{\lambda_{i}\right\}$ with $\lim _{i \rightarrow \infty} \lambda_{i}=0$, there exists a subsequence, still denoted by $\left\{\lambda_{i}\right\}$, a constant $\theta$, and a positive function $w$ such that

(1) $w$ is a solution of the problem

$$
\begin{gathered}
-\operatorname{div}\left(|\nabla u|^{p-2} \nabla u\right)=\theta L_{0} u^{q} \quad \text { in } \Omega, \\
u=0 \quad \text { on } \partial \Omega,
\end{gathered}
$$

(2) $\left\{u\left(\cdot, \lambda_{i}\right) /\left\|u\left(\cdot, \lambda_{i}\right)\right\|_{\infty}\right\}$ converges to $w$ in $C^{1}(\bar{\Omega})$ as $i \rightarrow \infty$.

To obtain Theorem 2.1, the following lemma is established:

LEMMA 2.2. Let $f$ satisfy condition $(\mathrm{A})$ and $u_{\lambda} \in C_{0}^{1}(\bar{\Omega})$ be a radially symmetric positive solution of (1.1)-(1.2). Then $\lim _{\lambda \rightarrow 0^{+}}\left\|u_{\lambda}\right\|_{\infty}=\infty$.

Proof. On the contrary, assume that there exist sequences $\left\{\lambda_{n}\right\}$ and $\left\{u_{n}\right\} \equiv\left\{u_{\lambda_{n}}\right\} \in C_{0}^{1}(\bar{\Omega})$ such that $\lambda_{n} \rightarrow 0$ and $\left\|u_{n}\right\| \leq M$, where $M>0$ is independent of $n$. Then $\left\|u_{n}\right\|_{\infty} \nrightarrow 0$ as $n \rightarrow \infty$. Indeed, suppose this does not hold; by the regularity of $-\operatorname{div}\left(|\nabla \cdot|^{p-2} \nabla \cdot\right)$ (see $\left.[6]\right)$, there exists $\omega \geq 0$ in $\Omega$ such that $\lambda_{n}^{-1 /(p-1)} u_{n} \rightarrow \omega$ in $C^{1}(\Omega)$ as $n \rightarrow \infty$. Moreover, $\omega$ satisfies the problem

$$
\begin{gathered}
-\operatorname{div}\left(|\nabla \omega|^{p-2} \nabla \omega\right)=f(0)<0 \quad \text { in } \Omega, \\
\omega=0 \quad \text { on } \partial \Omega .
\end{gathered}
$$


It follows from the maximum principle that $\omega<0$ in $\Omega$. This is impossible. Now, since $u_{n}$ is uniformly bounded in $\Omega$ and $\lambda_{n} \rightarrow 0$ as $n \rightarrow \infty$, it follows from the regularity of $-\operatorname{div}\left(|\nabla \cdot|{ }^{p-2} \nabla \cdot\right)$ again that there exists $\bar{\omega} \in C_{0}^{1}(\Omega)$ with $\bar{\omega} \geq 0$ in $\Omega$ such that $u_{n} \rightarrow \bar{\omega}$ in $C^{1}(\Omega)$ as $n \rightarrow \infty$ and $\bar{\omega}$ satisfies

$$
\begin{array}{cc}
-\operatorname{div}\left(|\nabla \bar{\omega}|^{p-2} \nabla \bar{\omega}\right) \equiv 0 \quad \text { in } \Omega, \\
\bar{\omega}=0 \quad \text { on } \partial \Omega . &
\end{array}
$$

Thus, $\bar{\omega} \equiv 0$ in $\Omega$. This also implies that $u_{n} \rightarrow 0$ in $C^{1}(\Omega)$ as $n \rightarrow \infty$. But the above argument implies that this is impossible. Hence, we conclude that $\left\|u_{n}\right\|_{\infty} \rightarrow \infty$ as $n \rightarrow \infty$.

Lemma 2.3. Let $a>0$. Then, for any $\theta \leq 0$, the equation

$$
\left(\left|u^{\prime}\right|^{p-2} u^{\prime}\right)^{\prime}+a u(s)^{\mu}=0 \quad \text { in }(\theta, \infty)
$$

has no bounded positive solution $u \in C^{1}(\theta, \infty)$ with $u^{\prime}(0)=0$. Moreover, the equation

$$
\left(\left|u^{\prime}\right|^{p-2} u^{\prime}\right)^{\prime}+a u(s)^{\mu}=0 \quad \text { in }(-\infty, \infty)
$$

has no bounded positive entire solution $u \in C^{1}(-\infty, \infty)$ with $u^{\prime}(0)=0$.

Proof. Suppose that such a solution $u(s)$ exists. Let $\Phi_{p}(y)=|y|^{p-2} y$. Then

$$
\Phi_{p}\left(u^{\prime}(s)\right)=-\int_{0}^{s} a u(\xi)^{\mu} d \xi \quad \text { for } s \in(0, \infty) .
$$

Thus, $\Phi_{p}\left(u^{\prime}\left(s_{0}\right)\right)=-k<0$ for some $s_{0}>0$ where $k=a \int_{0}^{s_{0}} u(\xi)^{\mu} d \xi$. By $(2.2), \Phi_{p}\left(u^{\prime}(s)\right) \leq-k$ for $s>s_{0}$, since $u(s)>0$ for $s>0$. Then

$$
u^{\prime}(s) \leq \Phi_{p}^{-1}(-k)=-k^{1 /(p-1)} \quad \text { for } s>s_{0} .
$$

Integrating (2.3) over $\left(s_{0}, s\right)$, we obtain $u(s) \rightarrow-\infty$ as $s \rightarrow \infty$, contrary to the assumption that $u(s)$ is a bounded solution.

Proof of Theorem 2.1. By the standard estimates for elliptic equations and condition (B), it follows that

$$
\begin{aligned}
\|u(\cdot, \lambda)\|_{\infty}^{p-1} & \leq C(\Omega) \lambda\|f(u(\cdot, \lambda))\|_{\infty} \\
& =C(\Omega) \lambda\left\|L_{0} u(\cdot, \lambda)^{q}+\left\{f(u(\cdot, \lambda))-L_{0} u(\cdot, \lambda)^{q}\right\}\right\|_{\infty} .
\end{aligned}
$$

That is,

$$
\begin{aligned}
1 \leq & C(\Omega) \lambda L_{0} \frac{\left\|u(\cdot, \lambda)^{q}\right\|_{\infty}}{\|u(\cdot, \lambda)\|_{\infty}^{p-1}} \\
& +C(\Omega) \lambda\left\|\frac{f(u(\cdot, \lambda))-L_{0} u(\cdot, \lambda)^{q}}{u(\cdot, \lambda)^{q}+1}\right\|_{\infty} \frac{\left\|u(\cdot, \lambda)^{q}+1\right\|_{\infty}}{\|u(\cdot, \lambda)\|_{\infty}^{p-1}} .
\end{aligned}
$$


By (B), there exists a positive constant $K_{0}$ such that

$$
\left|\left(f(u)-L_{0} u^{q}\right) /\left(u^{q}+1\right)\right|<K_{0} \quad \text { for } u \in \mathbb{R}^{+} .
$$

Then

$$
1 \leq C(\Omega) \lambda\|u(\cdot, \lambda)\|_{\infty}^{q-p+1}+C(\Omega) \lambda K_{0}\left\{\|u(\cdot, \lambda)\|_{\infty}^{q-p+1}+\frac{1}{\|u(\cdot, \lambda)\|_{\infty}^{p-1}}\right\} .
$$

From $\lim _{\lambda \rightarrow 0}\|u(\cdot, \lambda)\|_{\infty}=\infty$, it follows that there exists a positive constant $K_{1}$ such that, for any $\lambda \in\left(0, \lambda_{0}\right), K_{1}<\lambda\|u(\cdot, \lambda)\|_{\infty}^{q-p+1}$.

Thus, the left-hand inequality in Theorem 2.1 is established.

To obtain the other half of Theorem 2.1, we show that $T=\lambda\|u\|_{\infty}^{q-p+1}$ is bounded as $\lambda \rightarrow 0$. Let $u_{\lambda}$ be a positive radial solution of (1.1)-(1.2) satisfying $\left\|u_{\lambda}\right\|_{\infty} \rightarrow \infty$ as $\lambda \rightarrow 0^{+}$. Then there exists a positive solution $v_{\lambda}$ of (1.5)-(1.6) satisfying $\left\|v_{\lambda}\right\|_{\infty} \rightarrow \infty$ as $\lambda \rightarrow 0^{+}$. Let $\left(\lambda_{n}, v_{n}\right)$ be a positive solution of (1.5)-(1.6) with $\lambda=\lambda_{n}$ satisfying $\lambda_{n} \rightarrow 0^{+}$and $\left\|v_{n}\right\|_{\infty} \rightarrow \infty$ as $n \rightarrow \infty$. Then $w_{n}=v_{n} /\left\|v_{n}\right\|_{\infty}$ satisfies

$$
-\left(\Phi_{p}\left(w_{n}^{\prime}(s)\right)\right)^{\prime}=\lambda_{n}\left\|v_{n}\right\|^{q-p+1}\left(\frac{p-1}{N-p}\right)^{p} s^{-p(N-1) /(N-p)} \frac{f\left(v_{n}\right)}{\left\|v_{n}\right\|_{\infty}^{q}},
$$

and $w_{n}\left(s_{1}\right)=w_{n}\left(s_{2}\right)=0,\left\|w_{n}\right\|_{\infty}=1$.

Now, we show that $\left\{T_{n}\right\}=\left\{\lambda_{n}\left\|v_{n}\right\|_{\infty}^{q-p+1}\right\}$ is bounded. We prove this by a blowing up argument as in [3]. Suppose that $T_{n} \rightarrow \infty$ as $n \rightarrow \infty$. Let $\widehat{s}_{n} \in\left(s_{1}, s_{2}\right)$ be such that $w_{n}\left(\widehat{s}_{n}\right)=1, y_{n}=T_{n}^{1 / p}\left(s-\widehat{s}_{n}\right)$ and $\widehat{w}_{n}\left(y_{n}\right)=$ $w_{n}(s)$. Then $\widehat{w}_{n}(0)=1, \widehat{w}_{n}^{\prime}(0)=0$ and $\widehat{w}_{n}\left(y_{n}\right)$ satisfies

$$
\begin{aligned}
-\left(\Phi_{p}\left(\widehat{w}_{n}^{\prime}\right)\right)^{\prime}= & \left(\frac{p-1}{N-p}\right)^{p}\left(y_{n} T_{n}^{-1 / p}+\widehat{s}_{n}\right)^{-p(N-1) /(N-p)} \\
& \times \frac{f\left(\left\|v_{n}\right\|_{\infty} \widehat{w}_{n}\left(y_{n}\right)\right)}{\left\|v_{n}\right\|_{\infty}^{q}} .
\end{aligned}
$$

Since $\widehat{s}_{n} \in\left[s_{1}, s_{2}\right]$ and $f(s) \leq \beta_{1}+\beta_{2} s^{q}$ and $\left\|v_{n}\right\|_{\infty} \rightarrow \infty$ as $n \rightarrow \infty$, the right-hand side of (2.5) is uniformly bounded. Thus, there exist subsequences (still denoted by $\left\{\widehat{s}_{n}\right\},\left\{\widehat{w}_{n}\right\}$ and $\left.\left\{v_{n}\right\}\right)$ such that $\widehat{w}_{n} \rightarrow \widehat{w}$ in $C_{\text {loc }}^{1}(-\infty, \theta)$ (or $C_{\text {loc }}^{1}(-\infty, \infty)$ or $C_{\text {loc }}^{1}(\theta, \infty)$ ) as $n \rightarrow \infty$. Here $\theta \leq 0$ is a fixed number since the limit of $\widehat{s}_{n}$ may be $s_{1}$ or $s_{2}$ and $T_{n} \rightarrow \infty$. If $\widehat{s}_{n} \rightarrow s_{1}$ as $n \rightarrow \infty$, we assume that $\lim _{n \rightarrow \infty} T_{n}^{1 / p}\left(s_{1}-\widehat{s}_{n}\right)=\theta \leq 0$ (or $\left.\theta=-\infty\right)$. Otherwise, we can choose a subsequence of $\left\{T_{n}^{1 / p}\left(s_{1}-\widehat{s}_{n}\right)\right\}$ whose limit exists (or is $\left.-\infty\right)$. If the limit of $\widehat{s}_{n}$ is $s_{2}$, and if we set $y_{n}=T_{n}^{1 / p}\left(\widehat{s}_{n}-s_{2}\right)$, it follows that $\widehat{w}_{n} \rightarrow \widehat{w}$ in $C_{\text {loc }}^{1}(-\infty, \infty)$ (or $\left.C_{\text {loc }}^{1}(\theta, \infty), \theta \leq 0\right)$ as $n \rightarrow \infty$. Therefore, we assume that $\widehat{w}_{n} \rightarrow \widehat{w}$ in $C_{\text {loc }}^{1}(\theta, \infty)$ (or $\left.C_{\text {loc }}^{1}(-\infty, \infty)\right)$ ). Since $\widehat{w} \in C^{1}(\theta, \infty)$ (or $\left.C^{1}(-\infty, \infty)\right)$ satisfies $-\left(\Phi_{p}\left(\widehat{w}^{\prime}\right)\right)^{\prime} \geq 0$ in $(\theta, \infty)$ (or $\left.(-\infty, \infty)\right)$, and $\widehat{w}(0)=1$ and $\widehat{w}^{\prime}(0)=0$, the strong maximum principle as in Lemma 2.3 of [6] implies that $\widehat{w}>0$ in $(\theta, \infty)$ (or $(-\infty, \infty))$. Thus, for any interval in $(\theta, \infty)$ (or 
$(-\infty, \infty))$, there exists an $\omega>0$ such that $\widehat{w}(x)>\omega$ in this interval. This implies that

$$
\frac{f\left(\left\|v_{n}\right\|_{\infty} \widehat{w}_{n}\right)}{\left\|v_{n}\right\|_{\infty}^{q}} \rightarrow L_{0} \widehat{w}^{q}
$$

in $C_{\text {loc }}(\theta, \infty)$ (or $\left.C_{\text {loc }}(-\infty, \infty)\right)$ as $n \rightarrow \infty$. Therefore, $\widehat{w}$ satisfies

$$
-\left(\Phi_{p}\left(\widehat{w}^{\prime}\right)\right)^{\prime}=L_{0}((p-1) /(N-p))^{p} s_{*}^{-p(N-1) /(N-p)} \widehat{w}^{q}
$$

in $(\theta, \infty)($ or $(-\infty, \infty))$. Here $s_{*}=\lim _{n \rightarrow \infty} \widehat{s}_{n}$. This contradicts Lemma 2.3. Thus, $\left\{T_{n}\right\}$ is bounded. Therefore

$$
K_{1}<\lambda\left\|u_{\lambda}\right\|_{\infty}^{q-p+1}<K_{2} .
$$

Finally, let $\left\{\lambda_{i}\right\}$ be a sequence with $\lim _{i \rightarrow \infty} \lambda_{i}=0$ and denote the quantity $\lambda_{i}\left\|v\left(\cdot, \lambda_{i}\right)\right\|_{\infty}^{q-p+1}$ by $\theta_{i}$. Suppose that $\theta \in\left[K_{1}, K_{2}\right]$ is any accumulation point of $\left\{\theta_{i}\right\}$. Thus there exists a subsequence of $\left\{\theta_{i}\right\}$ (still denoted by $\left\{\theta_{i}\right\}$ later) which converges to $\theta$. Let $w(x, \lambda)=v(x, \lambda) /\|v(\cdot, \lambda)\|_{\infty}$. Then $\|w(\cdot, \lambda)\|_{\infty}=1$ and

$$
-\operatorname{div}\left(|\nabla w|^{p-2} \nabla w\right)=\theta_{i} \frac{f\left(v\left(x, \lambda_{i}\right)\right)}{\left\|v\left(\cdot, \lambda_{i}\right)\right\|_{\infty}^{q}} .
$$

Using the same idea as above for (2.4), we find a function $w(\cdot)$ and a subsequence of $\left\{w\left(\cdot, \lambda_{i}\right)\right\}$ (still denoted by $\left.\left\{w\left(\cdot, \lambda_{i}\right)\right\}\right)$ such that $\left\{w\left(\cdot, \lambda_{i}\right)\right\}$ converges to $w$ in $C^{1}\left(s_{1}, s_{2}\right)$ as $i \rightarrow \infty$. By condition (B), it follows that

$$
\lim _{i \rightarrow \infty} \frac{f\left(\left\|v\left(\cdot, \lambda_{i}\right)\right\|_{\infty} w\left(x, \lambda_{i}\right)\right)}{\left\|v\left(\cdot, \lambda_{i}\right)\right\|_{\infty}^{q}}=L_{0} w^{q} .
$$

Therefore $w(\cdot)$ is a positive solution of the problem

$$
\begin{gathered}
-\operatorname{div}\left(|\nabla w|^{p-2} \nabla w\right)=\theta L_{0} w^{q} \quad \text { in } \Omega, \\
w=0 \quad \text { on } \partial \Omega,
\end{gathered}
$$

and $\|w(\cdot)\|_{\infty}=1$.

3. A priori estimates for $\Omega$ being a ball. In this section, consider the set of radially symmetric positive solutions to the equation

$$
\begin{gathered}
-\operatorname{div}\left(|\nabla u|^{p-2} \nabla u\right)=\lambda f(u) \quad \text { for } x \in \Omega, \\
\left.u\right|_{\partial \Omega}=0,
\end{gathered}
$$

where $\Omega$ denotes the unit ball in $\mathbb{R}^{N}(N>1)$, centered at the origin, and $\lambda>0$. Here $f:[0, \infty) \rightarrow \mathbb{R}$ is assumed to satisfy

$$
f(0)<0 \text { (non-positone), } f^{\prime}(u) \geq 0 \text {, and } f\left(u_{0}\right)>0 \text { for some } u_{0}>0 .
$$

Let $F$ be defined as $F(t)=\int_{0}^{t} f(s) d s$, and let $\beta$ and $\theta(\beta<\theta)$ be the unique positive zeros of $f$ and $F$, respectively.

In this section, the following theorem is proved: 
THEOREM 3.1. Let $u$ be a radially symmetric positive solution of (3.1)(3.2) with $u(0)=d$ and suppose $f$ satisfies (3.3). Then for large $\lambda$,

$$
\begin{aligned}
\left(\frac{p}{p-1}\right)^{p-1}(N-1) & \leq \frac{\lambda f(d)}{d^{p-1}} \\
& \leq \frac{2 N f(d)}{d^{p-1}}\left(\frac{p}{p-1}\right)^{p-1}\left(\int_{\theta}^{d} \frac{d s}{f(s)^{1 /(p-1)}}\right)^{p-1} .
\end{aligned}
$$

REMARK. If $f(u) \leq M$ for all $u$, or if $f(u)=u^{\alpha}-1$ where $0<\alpha<p-1$, then $f(d) d^{-(p-1)}\left(\int_{\theta}^{d} f(s)^{-1 /(p-1)} d s\right)^{p-1}$ is finite.

Note that radially symmetric positive solutions of (3.1)-(3.2) are strictly decreasing in $r$ for $r \in(0,1)$ where $r=\|x\|$. Thus, they satisfy

$$
\begin{gathered}
\left(\Phi_{p}\left(u^{\prime}\right)\right)^{\prime}+\frac{N-1}{r} \Phi_{p}\left(u^{\prime}\right)+\lambda f(u)=0 \quad \text { in }(0,1), \\
u(0)=d, \quad u^{\prime}(0)=0, \quad u(1)=0, \quad u^{\prime}(r)<0 \quad \text { in }(0,1) .
\end{gathered}
$$

where $\Phi_{p}(s)=|s|^{p-2} s, p>1$.

If $u$ is a solution of (3.5)-(3.6), then multiplying (3.5) by $r^{N-1}$ and integrating from 0 to $t$ gives

$$
-\int_{0}^{t}\left(r^{N-1} \Phi_{p}\left(u^{\prime}\right)\right)^{\prime} d r=\int_{0}^{t} \lambda r^{N-1} f(u) d r .
$$

Since $u$ is decreasing and $f$ is increasing, it follows that

$$
-t^{N-1} \Phi_{p}\left(u^{\prime}\right)=\lambda \int_{0}^{t} r^{N-1} f(u) d r \geq \lambda f(u(t)) \int_{0}^{t} r^{N-1} d r=\frac{\lambda t^{N-1} f(u)}{N} .
$$

Hence

$$
\left(-u^{\prime}\right)^{p-1} \geq \frac{\lambda t f(u)}{N}
$$

Next, multiplying (3.5) by $u^{\prime}$ and integrating over $[0,1]$ yields

$$
\frac{p-1}{p}\left|u^{\prime}(1)\right|^{p}+\int_{0}^{1} \frac{N-1}{r}\left|u^{\prime}\right|^{p} d r=\lambda F(d) .
$$

Note that this implies

$$
d>\theta .
$$

To prove Theorem 3.1, we need the following lemma:

Lemma 3.2 (see [19]). Let $u$ be a radially symmetric positive solution of (3.1)-(3.2). Then there exists $M>0$ such that for large $\lambda$,

$$
\left|u^{\prime}(1)\right|>\lambda^{1 /(p-1)} M \text {. }
$$


The proof of Theorem 3.1 is based upon a modification of the method of Iaia [12].

Proof of Theorem 3.1. First, Hölder's inequality gives

$$
\begin{aligned}
d & =u(0)-u(1)=-\int_{0}^{1} u^{\prime}(t) d t=\int_{0}^{1} \frac{-u^{\prime}}{t^{1 / p}} t^{1 / p} d t \\
& \leq\left(\int_{0}^{1} \frac{\left|u^{\prime}\right|^{p}}{t} d t\right)^{1 / p}\left(\int_{0}^{1} t^{1 /(p-1)} d t\right)^{(p-1) / p} .
\end{aligned}
$$

Next, it follows from (3.8) that

$$
d^{p} \leq\left(\frac{p-1}{p}\right)^{p-1} \int_{0}^{1} \frac{\left|u^{\prime}\right|^{p}}{t} d t \leq\left(\frac{p-1}{p}\right)^{p-1} \frac{\lambda F(d)}{N-1} .
$$

Thus

$$
\frac{\lambda F(d)}{d^{p}} \geq\left(\frac{p}{p-1}\right)^{p-1}(N-1) .
$$

Finally, since $f^{\prime} \geq 0$,

$$
F(d)=\int_{0}^{d} f(s) d s=d f(d)-\int_{0}^{d} s f^{\prime}(s) d s \leq d f(d) .
$$

This proves the left-hand inequality of (3.4).

In order to establish the right-hand inequality of (3.4), from (3.7) we get

$$
-u^{\prime}(t) \geq\left(\frac{\lambda t f(u)}{N}\right)^{1 /(p-1)} .
$$

Let $q_{\lambda} \in(0,1)$ be such that $u\left(q_{\lambda}\right)=\theta$. Then $u(t) \geq \theta>\beta$ on $\left[0, q_{\lambda}\right]$. Thus $f(u(t)) \geq f(\theta)>f(\beta)=0$ on $\left[0, q_{\lambda}\right]$. So, on $\left[0, q_{\lambda}\right]$ we have

$$
\int_{0}^{q_{\lambda}} \frac{-u^{\prime}}{f(u)^{1 /(p-1)}} d t \geq \int_{0}^{q_{\lambda}}\left(\frac{\lambda t}{N}\right)^{1 /(p-1)} d t=\left(\frac{\lambda}{N}\right)^{1 /(p-1)}\left(\frac{p-1}{p}\right) q_{\lambda}^{p /(p-1)} .
$$

Changing variables in the first integral via $s=u(t)$ gives

$$
\int_{\theta}^{d} \frac{d s}{f(s)^{1 /(p-1)}} \geq\left(\frac{\lambda}{N}\right)^{1 /(p-1)}\left(\frac{p-1}{p}\right) q_{\lambda}^{p /(p-1)} .
$$

Thus,

$$
\frac{f(d)^{1 /(p-1)}}{d} \int_{\theta}^{d} \frac{d s}{f(s)^{1 /(p-1)}} \geq \frac{(\lambda f(d))^{1 /(p-1)}}{N^{1 /(p-1)} d}\left(\frac{p-1}{p}\right) q_{\lambda}^{p /(p-1)} .
$$

Therefore, the proof of Theorem 3.1 will be completed once the following lemma is established. 
LEMMA 3.3. $\lim _{\lambda \rightarrow \infty} q_{\lambda}=1$.

From this lemma, for large $\lambda$, we have $q_{\lambda}^{p} \geq 1 / 2$. Substituting this into (3.11), one can deduce

$$
\frac{\lambda f(d)}{d^{p-1}} \leq \frac{2 N f(d)}{d^{p-1}}\left(\frac{p-1}{p}\right)^{p-1}\left(\int_{\theta}^{d} \frac{d s}{f(s)^{1 /(p-1)}}\right)^{p-1},
$$

which completes the proof of Theorem 3.1.

Proof of Lemma 3.3. Multiplying (3.5) by $u^{\prime}$ and integrating from $t$ to 1 gives

$$
\int_{t}^{1}\left[u^{\prime}\left(\Phi_{p}\left(u^{\prime}\right)\right)^{\prime}+\frac{N-1}{r}\left|u^{\prime}\right|^{p}\right] d r=\int_{t}^{1}\left(-\lambda f(u) u^{\prime}\right) d r .
$$

Thus

$$
\frac{p-1}{p}\left[\left|u^{\prime}\right|^{p}(1)-\left|u^{\prime}\right|^{p}(t)\right]+\int_{t}^{1} \frac{N-1}{r}\left|u^{\prime}\right|^{p} d r=-\lambda[F(u(1))-F(u(t))] .
$$

Since $F(u(1))=F(0)=0$, it follows that

$$
\frac{p-1}{p}\left[\left|u^{\prime}\right|^{p}(1)-\left|u^{\prime}\right|^{p}(t)\right] \leq \lambda F(u(t)) .
$$

Now, for $q_{\lambda} \leq t \leq 1$, it follows that $\theta=u\left(q_{\lambda}\right) \geq u(t) \geq u(1)=0$, and then $F(u(t)) \leq 0$. Hence,

$$
\left|u^{\prime}\right|^{p}(1) \leq\left|u^{\prime}\right|^{p}(t) \quad \text { for } t \in\left[q_{\lambda}, 1\right] .
$$

Now Lemma 3.2 shows that there exists a $c>0$ independent of $\lambda$ such that

$$
-u^{\prime}(1) \geq c \lambda^{1 /(p-1)} \quad \text { for large } \lambda .
$$

Consequently, it follows from (3.12) that

$$
\left(-u^{\prime}(t)\right)^{p} \geq\left(-u^{\prime}(1)\right)^{p} \geq c^{p} \lambda^{p /(p-1)} \quad \text { for } t \in\left[q_{\lambda}, 1\right] .
$$

Integrating on $\left[q_{\lambda}, 1\right]$ gives

$$
\theta=u\left(q_{\lambda}\right)=-\int_{q_{\lambda}}^{1} u^{\prime}(t) d t \geq \int_{q_{\lambda}}^{1} c \lambda^{1 /(p-1)} d t=c \lambda^{1 /(p-1)}\left(1-q_{\lambda}\right) .
$$

Thus

$$
0 \leq 1-q_{\lambda} \leq \frac{\theta}{c \lambda^{1 /(p-1)}} .
$$

As $\lambda \rightarrow \infty$ the right-hand side of the above expression tends to zero; hence $\lim _{\lambda \rightarrow \infty} q_{\lambda}=1$ and this completes the proof of the lemma. 


\section{References}

[1] J. I. Diaz, Nonlinear Partial Differential Equations and Free Boundaries, Vol. I, Elliptic Equations, Res. Notes in Math. 106, Pitman, London, 1985.

[2] S. Fučik, J. Nečas, J. Souček and V. Souček, Spectral Analysis of Nonlinear Operators, Lecture Notes in Math. 346, Springer, Berlin, 1973.

[3] B. Gidas and J. Spruck, A priori bounds for positive solutions of nonlinear elliptic equations, Comm. Partial Differential Equations 6 (1981), 883-901.

[4] M. Guedda and L. Véron, Local and global properties of quasilinear elliptic equations, J. Differential Equations 76 (1988), 159-189.

[5] Z. M. Guo, Existence and uniqueness of the positive radial solutions for a class of quasilinear elliptic equations, Appl. Anal. 47 (1992), 173-190.

[6] - Some existence and multiplicity results for a class of quasilinear elliptic equations, Nonlinear Anal. 18 (1992), 957-971.

[7] - On the positive solutions for a class of quasilinear non-positone problems, Chinese Quart. J. Math. 1996, 1-11.

[8] Z. M. Guo and J. R. L. Webb, Uniqueness of positive solutions for quasilinear elliptic equations when a parameter is large, Proc. Roy. Soc. Edinburgh Sect. A 124 (1994), 189-198.

[9] Z. M. Guo and Z. D. Yang, Structure of positive solutions to a class of quasilinear elliptic equations with a small parameter, Chinese Ann. Math. Ser. A 19 (1998), 385-392.

[10] - - - Some uniqueness results for a class of quasilinear elliptic eigenvalue problems, Acta Math. Sinica 14 (1998), 245-260.

[11] J. A. Iaia, A priori estimates for a semilinear elliptic PDE, Nonlinear Anal. 24 (1995), 1039-1048.

[12] - A priori estimates and uniqueness of inflection points for positive solutions of semipositone problems, Differential Integral Equations 8 (1995), 393-403.

[13] W. M. Ni and J. Serrin, Nonexistence theorems for singular solutions of quasilinear partial differential equations, Comm. Pure Appl. Math. 39 (1986), 379-399.

[14] M. del Pino, M. Elgueta and R. Manásevich, A homotopic deformation along $p$ of a Leray-Schauder degree result and existence for $\left(\left|u^{\prime}\right|^{p-2} u^{\prime}\right)^{\prime}+f(t, u)=0, u(0)=$ $u(T)=0, p>1$, J. Differential Equations 80 (1989), 1-13.

[15] P. Tolksdorf, Regularity of a more general class of quasilinear elliptic equations, ibid. 51 (1984), 126-150.

[16] Z. D. Yang, Existence of positive solutions for a class of singular two-point boundary value problems of second order nonlinear equations, Appl. Math. Mech. 17 (1996), 465-476.

[17] -, Non-existence of positive entire solutions for elliptic inequalities of p-Laplacian, Appl. Math. J. Chinese Univ. Ser. B 12 (1997), 399-410.

[18] Z. D. Yang and Z. M. Guo, On the structure of positive solutions for quasilinear ordinary differential equations, Appl. Anal. 58 (1995), 31-51.

[19] Z. D. Yang, X. H. Wu and H. S. Yang, A priori estimates of positive radially symmetric solutions for quasilinear elliptic equations, Math. Appl. 12 (1999), no. 4, $1-4$.

[20] Z. D. Yang and H. S. Yang, Non-negative solutions for a class of non-positone problems, Ann. Differential Equations 13 (1997), 304-312. 
[21] Z. D. Yang and H. S. Yang, A priori estimates for a quasilinear elliptic partial differential equations non-positone problems, Nonlinear Anal. 43 (2001), 173-181.

College of Science

Beijing University of Aeronautics and Astronautics

Beijing, China, 100083

E-mail: zdyang_jin@263.net 